\section{PERFILES PEDAGÓGICOS DEL PROFESORADO DE EDUCACIÓN FÍSICA EN UNA ESCUELA MULTICULTURAL}

\author{
PERFIS PEDAGÓGICOS DE PROFESSORES DE EDUCAÇÃO FÍSICA EM UMA \\ ESCOLA MULTICULTURAL
}
PEDAGOGICAL PROFILES OF PHYSICAL EDUCATION TEACHERS IN A MULTICULTURAL SCHOOL

Gonzalo Flores Aguilar*, Maria Prat Grau**, Susanna Soler Prat ${ }^{\star * *}$

\author{
Gonzalo Flores Aguilat,
}

Palabras clave: Educación Física. Competencia cultural.

Profesores.

Inmigrantes.

\begin{abstract}
Resumen: Este trabajo presenta una tipificación de los perfiles pedagógicos del profesorado de Educación Física en escuelas multiculturales a partir de sus percepciones, su intervención pedagógica y su trayectoria profesional. Para ello, se ha realizado un estudio de casos múltiple, con doce docentes de escuelas públicas de Barcelona con más del $50 \%$ de alumnado inmigrante. Las técnicas de investigación han sido la entrevista semiestructurada y la observación no participante, junto con el correspondiente análisis de contenido. Los datos recogidos, analizados desde la óptica intercultural, han permitido establecer tres perfiles docentes: el "sensible-inclusor", el "romántico-asimilador" y el "quemado-prejuicioso". Esta tipificación permite reconocer la diversidad de posturas existentes entre el colectivo profesional y analizar las necesidades formativas y de apoyo más oportunas en cada caso.
\end{abstract}

Palavras chave: Educação Física. Competência cultural. Professores escolares. Imigrantes.

Keywords: Physical Education. Cultural Competence. School Teachers. Immigrants.
Resumo: Este artigo apresenta uma classificação dos perfis pedagógicos dos professores de Educação Física de escolas multiculturais a partir de suas próprias percepções, de sua intervenção pedagógica e de sua trajetória profissional. Foi realizado um estudo de casos múltiplos com 12 professores que trabalham em escolas públicas de Barcelona, com mais de $50 \%$ de estudantes imigrantes. As técnicas de investigação utilizadas foram entrevistas semiestruturadas e observações não participantes, juntamente com a Análise de Conteúdo correspondente. Os dados recolhidos a partir da ótica intercultural permitiram estabelecer três perfis docentes: o "sensível inclusivo", o "assimilador romântico" e 0 "queimado-preconceituoso". Essa classificação permite reconhecer a diversidade de posturas existentes nesse coletivo profissional e analisar as necessidades formativas e de suporte mais oportunas em cada caso.

Abstract: This paper presents a categorization of pedagogical profiles of Physical Education teachers in multicultural schools, based on their perceptions, pedagogical interventions and professional histories. A multiple case study was conducted with twelve teachers from public schools in Barcelona where over $50 \%$ of students were immigrants. The study used semi-structured interviews and non-participant observation, and then conducted the corresponding content analysis. The data collected were analysed from the cross-cultural point of view, and three teacher profiles were established: "sensitiveinclusive"; "romantic-assimilator"; and "stressed out-prejudiced". This classification allowed us to recognize the diversity of attitudes among these professionals and to analyze the most appropriate training and support needs for each case..
*Universidad de Barcelona; Universidad de Vic-Universidad Central del Cataluña. Barcelona, España. E-mail: gonzalo.flores@ub.edu; gonzalo.flores@uvic.cat

**Universidad Autónoma de Barcelona. Barcelona, España.

E-mail: maria.prat@uab.cat

***Instituto Nacional de Educación Física de Cataluña (INEFC). Barcelona, España.

E-mail: ssoler@gencat.cat

Recebido em: 20-04-2018 Aprovado em: 29-03-2019 Publicado em: 03-05-2019

DOI: https://doi.org/10.22456/1982-8918.82139 (c) (i) () ${ }_{\mathrm{BY}}^{\text {Licence }}$ Creative Commom 


\section{INTRODUCCIÓN}

En esta última década, España se reafirma como un país receptor de población inmigrada (EUROPEAN STATISTICS, 2017), y Cataluña como el territorio nacional que más acoge, con un 13,78\% de la población en 2017 (INSTITUT D'ESTADÍSTICA DE CATALUNYA, 2017). Esta realidad social genera nuevas oportunidades de enriquecimiento cultural, pero también supone un reto para la convivencia y la cohesión (FLECHA; PUIGVERT, 2002).

En este marco, las escuelas son un espacio clave por su rol inclusivo y transformador de la realidad social (SALES, 2007). Para ello, el modelo de Educación Intercultural (EI) se considera el enfoque más idóneo para cubrir las necesidades de la sociedad del siglo XXI (BESALÚ; LÓPEZ-CUESTA, 2011). No obstante, la atención educativa que actualmente recibe el alumnado inmigrante se basa en modelos segregacionistas y compensatorios (AGUADO; MATA; GIL-JAURENA, 2017) que contribuyen a la reproducción de la marginación y fomentan el fracaso escolar (GARRETA, 2011).

En base a los estudios analizados, este panorama es la consecuencia de las numerosas carencias existentes, como por ejemplo:

a) la ausencia de unas sólidas bases teóricas respecto a la El (AGUADO, 2003);

b) los insuficientes estudios empíricos y el carácter cuantitativo de los mismos; y

c) las dificultades del profesorado, incluyéndose al de Educación Física (EF), a la hora de abordar la El y la atención al alumnado inmigrante (FERNÁNDEZ-BATANERO; AGUILAR, 2016).

Por ello, y de cara a avanzar hacia el futuro de la Educación Física Intercultural (EFI) (FLORES; PRAT; SOLER, 2015a), el análisis de la realidad profesional y personal del profesorado de EF adquiere una especial relevancia ya que el profesorado es el principal agente de cambio (CHEPYATOR-THOMSON; YOU; RUSELL, 2000), y sus actuaciones pedagógicas pueden ser la clave para impulsar los principios interculturales (GIROUX, 1985). Además, la EF puede ser una de las mejores herramientas para atender al alumnado inmigrante y favorecer así la interculturalidad (CARTER; LÓPEZ-PASTOR; GALLARDO, 2017; SOLER; FLORES; PRAT, 2012), si bien son pocos los estudios realizados (CHOI; CHEPYATOR-THOMSON, 2011; LÓPEZ-PASTOR, 2012).

Aún así, varios trabajos internacionales alertan que la formación inicial y permanente que tradicionalmente ha recibido y recibe profesorado de EF sobre la atención a la diversidad cultural y la El es escasa y deficitoria (CHOI; CHEPYATOR-THOMSON, 2011; FLORES; PRAT; SOLER, 2014; GRIMINGER, 2011). Según Flores, Prat y Soler (2014), para una formación verdaderamente intercultural sería necesario:
a) reducir el etnocentrismo del currículum;
b) realizar una formación actitudinal;
c) fomentar un espíritu crítico y reflexivo;
d) favorecer la interacción y contacto con otras culturas; y
e) mejorar las competencias pedagógicas. 
A partir de estas consideraciones, este trabajo recoge la voz y la experiencia del día a día del profesorado de EF en contextos multiculturales. Concretamente, se pretende dar respuesta a las siguientes preguntas:

a) ¿cómo percibe la presencia del alumnado inmigrante en su aula?;

b) ¿cómo es su intervención pedagógica en un aula multicultural?; y

c) ¿cómo se ha desarrollado su trayectoria profesional en este contexto? A partir del análisis de estas tres dimensiones, y las bases teóricas de la El (AGUADO, 2003), se propone una tipificación de tres perfiles docentes. Estos perfiles deben ser entendidos como "tipos ideales" según el modo como fueron descritos por Weber (1983). Se trata, pues, de abstracciones 0 polarizaciones que permiten dar cuenta de una realidad.

Esta tipificación permite reconocer la diversidad de posturas existentes entre el colectivo profesional y analizar las necesidades formativas y de apoyo más oportunas en cada caso. En definitiva, con la descripción de estos perfiles se busca también conocer la manera de ser y actuar de los docentes en función de la realidad comentada; analizar sus características y los recursos utilizados en las aulas, o identificar carencias y déficits, para así profundizar en las problemáticas existentes y la forma de abordarlas en la formación inicial y permanente del profesorado.

\section{METODOLOGÍA}

Desde un enfoque cualitativo, se ha llevado a cabo un estudio de casos múltiple en el que han participado doce docentes (siete mujeres y cinco hombres), de diez escuelas diferentes. Los casos se han seleccionado en la ciudad de Barcelona y alrededores mediante un muestreo intencional (HEINEMANN, 2003), a partir de una serie de criterios: a) centros públicos de educación primaria con más del $50 \%$ de alumnado inmigrante; y b) profesorado de EF con $\geq$ cinco años de experiencia en dicha escuela.

Para la recogida de información se ha utilizado la entrevista semiestructurada y las notas de campo mediante observación no participante. Para los procesos de codificación, análisis e interpretación de los resultados se han aplicado las fases del análisis de contenido propuestas por Bardin (1986). A partir de las directrices de Lincoln y Guba (1985) sobre los criterios de rigurosidad de los datos, los hallazgos de este trabajo se consideran creíbles, transferibles y confiables, tal y como se desprenden de los ciclos reflexivos entre las personas investigadoras y los diversos agentes externos (miembros de los tribunales de seguimiento de la investigación; profesorado extranjero externo, etc.).

\section{RESULTADOS Y DISCUSIÓN}

A partir de los rasgos que caracterizan la Educación Intercultural y los aspectos surgidos en el análisis de las tres dimensiones estudiadas, a continuación se describen cada uno de los tres perfiles teóricos del profesorado de EF en escuelas multiculturales: el "sensible-inclusor", el "romántico-asimilador" y el "quemado-prejuicioso". Es importante destacar que se trata de perfiles teóricos, es decir, no hay un profesor o profesora (se utilizan pseudónimos) que encaje completamente en uno de estos perfiles. Es decir, podría decirse que son utópicos, aunque la mayoría de sus características definitorias si han podido ser identificadas, en mayor o menor escala, en esta investigación. 


\subsection{EL PERFIL "SENSIBLE-INCLUSOR"}

Este perfil pedagógico se caracteriza por disponer de una alta capacidad reflexiva y crítica, cualidad que se considera especialmente necesaria para la promoción de una El (BARKER, 2017). En su día a día, muestra inquietud para la transformación y mejora de la realidad social, se cuestiona sus propias creencias y actitudes, y reflexiona continuamente sobre el tipo y calidad de sus intervenciones en el aula

Tal y como también se describe en Capllonch, Godall y Lleixà (2007), desde este perfil se reconoce que la escolarización del alumnado inmigrante ha influido en su labor profesional, pero considera que no afecta negativamente: "¿Que es una dificultad? en algunos aspectos sí, pero le sacamos provecho" (entrevista con Darío).

Se concibe la diversificación cultural del alumnado como una oportunidad para educar hacia la tolerancia y convivencia entre culturas, adoptando el "enfoque dialógico" descrito por Flecha y Puigvert (2002): "Generalmente se vive de forma enriquecedora, tanto a nivel de convivencia como de aprender cosas nuevas de cada cultura en contacto" (entrevista con Rodrigo).

A su vez, y como recogen Benn y Dagkas (2006), desde este perfil se considera que el contacto directo que se crea en un aula multicultural entre el alumnado de diferentes orígenes es un aspecto positivo que favorece una mayor competencia intercultural, sobre todo en comparación con entornos más monoculturales. Por ello, valora la posibilidad de crecer juntos ya que permite reducir los prejuicios existentes (GARCÍA-FERNÁNDEZ; GOENECHEA, 2009). "Los niños de sexto (11-12 años) no están tan acostumbrados a la multiculturalidad y les cuesta más aceptar a una persona nueva" (entrevista con Mireia).

El profesorado pone de relieve que en las clases de EF se pueden resaltar las semejanzas entre las diversas culturas por encima de las diferencias. En este sentido, el profesorado se sitúa próximo a los planteamientos de Carbonell (2000), el cual señala la importancia de destacar que somos más iguales que diferentes.

Finalmente, otra característica clave de este perfil es la sensibilidad hacia las necesidades personales y educativas de su alumnado desde la perspectiva interseccional que proponen Flintoff, Fitzgerald y Scraton (2008). Es decir, tienen en cuenta no solo la diversidad cultural, sino también la diversidad de género y la condición socio-económica, tal y como recomiendan Besalú y Tort (2009).

\footnotetext{
Ya no es que culturalmente sean diferentes, porque eso no llega a ser un problema. Tienes que saber la problemática que estos niños llevan detrás. Hay niños que viven en un piso de Barcelona, con diez personas y camas calientes. Y ese niño, ¿qué haces con él? Es un poco complicado. (entrevista con Lorena).
}

De acuerdo con esta percepción, la intervención pedagógica del perfil "sensibleinclusor" guarda una estrecha relación con las características del "tacto pedagógico" de Van Manen (1998), ya que la premisa principal es la preocupación por el bien pedagógico de todos sus estudiantes. En la acogida del alumnado recién llegado, por ejemplo, utiliza diversas estrategias:

a) mostrar proximidad durante las sesiones mostrándose accesible y atento al estudiante, dándole confianza;

b) la realización de diferentes actividades de presentación; y 
c) el conocimiento previo de cada alumno o alumna antes de que acuda por primera vez a EF: "Yo intento entrar en el aula de acogida, conocerles antes de que vengan a la clase de EF para presentarme, hablar con ellos un poquito... para que también pierdan un poco el miedo." (entrevista con Álvaro). Según Oller y Colomé (2010), buena parte del éxito del enfoque intercultural y del futuro educativo del alumnado recién llegado pasa por el desarrollo de una adecuada acogida.

También se utilizan ciertas estrategias lingüísticas para favorecer la comunicación con el alumnado que desconoce las lenguas oficiales (catalán o castellano). Coincidiendo con las indicaciones de López-Pastor, Pérez y Monjas (2007), la figura del compañero/a intérprete es la más utilizada. Esta práctica tiene efectos muy positivos, tanto en la persona tutelada, como en la persona que ejerce el rol de tutor/a. "Una de las alumnas inmigrantes va siempre junto a su "compañera intérprete", que le ayuda a entender los ejercicios, le explica lo que dicen sus compañeros, y le sirve como medio para poder comunicarse con el resto de alumnos y con la profesora." (nota de campo de Lorena).

A su vez, utilizan también el inglés y/o algunas palabras clave en el idioma materno del alumnado: "Se ríen mucho porque digo palabras que suenan mal en chino. Se ríen, pero bueno, me parece una posibilidad que está bien." (entrevista con Hugo).

Goenechea, García-Fernández y Jiménez (2011), destacan como mediante esta estrategia los estudiantes pueden ver el "guiño" que estos profesionales dedican a su lengua, lo cual representa una valoración positiva hacia su cultura, con las ventajas emocionales que ello conlleva para el alumnado.

Por otra parte, este perfil también es consciente de las limitaciones de la EF y del lenguaje no verbal: "Hay niños que son más introvertidos y les da más vergüenza [...] hay niños que ni por imitación y que quizás llevan aquí tres o cuatro meses y aún están perdidos." (entrevista con Natalia).

Este perfil también se caracteriza por un fuerte vínculo afectivo-emocional con su alumnado, ya que lo considera un aspecto muy positivo para la escolarización del alumnado: "Lo único que quieren realmente estos niños es un poco de afectividad, y con eso ya lo has solucionado todo [...] aquí con los niños tienes un trato muy afectivo, demasiado. A veces haces más de mamá que no de profesora." (entrevista con Carmen).

Según Sampé, Arandia y Elboj (2012), estas relaciones son clave para facilitar la integración del alumnado en el sistema educativo. En esta línea, la comunicación con las familias procura ser fluida y permanente. El diálogo y la interacción con las familias es un principio básico para la implantación del enfoque intercultural en la escuela (GARCÍA-FERNÁNDEZ; GOENECHEA, 2009).

Yo hago mucho el "puerta a puerta", lo que llamamos aquí, el salir fuera de la escuela: "¿qué tal?, ¿cómo estás". Estableces unos vínculos que en un momento dado me pueden servir para conseguir cosas. Si yo cuido esas relaciones personales es mucho mejor para todos (entrevista con Hugo).

Por otra parte, desde este perfil, en el caso que haya alumnado que su tradición cultural o prácticas religiosas condicione su práctica, busca alternativas y realiza adaptaciones curriculares para que todo el alumnado pueda participar en las clases y actividades propuestas, coincidiendo con Carroll y Hollinshead (1993) y Dagkas (2007). 
La incorporación de los juegos o danzas del mundo en las programaciones a partir de las propias aportaciones del alumnado en la explicación y/o demostración de las actividades es también una práctica habitual: "Cuando el profesor ha dicho que iban a jugar a Baraf Pani uno de los alumnos procedentes de Pakistán se ha alegrado muy visiblemente. Entonces el profesor le ha propuesto que explique el juego al resto del grupo." (nota de campo de Rodrigo).

Soler y colaboradores (2012) subrayan que estas estrategias pueden ser beneficiosas ya que permiten aumentar su protagonismo de manera positiva en el aula, y pueden sentir valorada su cultura de origen. A su vez, y según el estadio aditivo propuesto por Banks (1995), el desarrollo curricular multi/inter-cultural necesita incorporar en el aula elementos pertenecientes a diferentes identidades étnicas para reducir la visión etnocéntrica del currículo, lo cual cobra una especial relevancia en un contexto culturalmente heterogéneo.

A partir de sus propios dilemas y necesidades, el perfil "sensible-inclusor" presenta un alto grado de preocupación por mejorar sus carencias formativas respecto a la atención del alumnado inmigrante y un gran interés por generar entornos y redes que le ayuden a desarrollar la El. Esto denota la existencia de un verdadero compromiso con su renovación pedagógica (MARCELO, 2008).

Para tal fin, y en coordinación con otros docentes del mismo barrio que comparten las mismas inquietudes sobre su labor profesional, el profesorado comparte y se forma al respecto.

Me he tenido que ir formando poco a poco. Siempre ha sido [la atención a la diversidad cultural] un eje vertebrador del seminario y grupo de trabajo del profesorado de EF de Ciutat Vella [distrito de Barcelona], que ya hace unos años que existe. Caminábamos de cara, sobre todo, a tratar la interculturalidad (entrevista con Rodrigo).

Este profesorado se caracteriza por presentar un buen estado de ánimo personal y profesional que, acompañado por un alto grado de ilusión y satisfacción laboral, favorece el deseo de continuar trabajando en su escuela actual. "Estoy súper contenta, súper feliz de estar aquí. Es que me llevo muy bien con los niños, yo disfruto, y ellos me enseñan cosas de sus países, y me cuentan historias de ellos." (entrevista con Lorena).

En definitiva, todas las características hasta ahora descritas del perfil "sensible-inclusor" nos permiten destacar a este perfil docente como el deseable para ser un agente de cambio que fomente la transformación social (BANKS; LYNCH, 1986).

\subsection{EL PERFIL "ROMÁNTICO-ASIMILADOR"}

Coincidiendo con los modelos educativos asimiladores y compensatorios (AGUADO; MATA; GIL-JAURENA, 2017), este perfil considera la EF como una panacea en la que la presencia de alumnado inmigrante no ha generado ningún tipo de cambio o problemática (FLORES; PRAT; SOLER, 2015b). La visión idealizada de los valores de la EF lleva al profesorado a percibir e interpretar la realidad educativa de forma utópica, en la que no hay ninguna situación a abordar. Este discurso romántico, que se genera por el amor incondicional hacia la EF, llega, pues a cegar al profesorado, tal y como describen Carroll y Hollinshead (1993).

Según este perfil docente, el alumnado inmigrante no ha provocado ningún problema 0 dificultad en las clases de EF ni en sus dinámicas de trabajo, sobre todo en comparación con 
las otras asignaturas que se imparten en el aula ordinaria. "Yo pienso que es mucho más difícil en un aula y no en EF porque dentro de un aula hay muchos desniveles, ya que está el que no sabe leer [...]. En EF solo juegan, son niños." (entrevista con Carmen).

El profesorado "romántico-asimilador" no duda en aludir y destacar a algunas de las principales singularidades y potencialidades de la EF y a ignorar sus limitaciones para la educación en valores y la inclusión (PRAT, 2010): "Yo creo que hay más dificultades en el aula que no en EF. En EF los niños te ayudan más, es una actividad más lúdica, están en un espacio abierto y no en un aula cerrada, donde muchas veces no entienden nada [...] el hábitat es diferente." (entrevista con Carmen).

Por otra parte, este perfil no percibe el carácter uniformizador y asimilador que a menudo se da en las actividades propuestas en EF y las dificultades que también genera el lenguaje corporal o las diferentes formas de ver y vivir el cuerpo y la actividad física en los diferentes marcos culturales (SOLER; ISPIZUA; MENDOZA, 2017). Lejos de cuestionar el enfoque etnocéntrico y androcéntrico de la $E F$, el profesorado reproduce la tradicional enseñanza de la materia, sin un posicionamiento personal crítico al respecto.

Desde este posicionamiento, existe un especial interés a la hora de subrayar que la presencia del alumnado inmigrante no conlleva ninguna modificación o revisión de la programación curricular, una idea que contradice a los consejos de López-Rodríguez y Sentís (2000): "Cambiar programaciones o maneras de hacer porque haya multiculturalidad, aquí no." (entrevista con Mireia).

A su vez, y coincidiendo con los resultados de Pastor, González, Cuevas y Gil-Madrona (2010), desde este perfil se afirma que tampoco es necesario llevar a cabo ningún tipo de adaptación curricular en el área de EF: "La naturaleza de mi asignatura ayuda a no tener que adaptar nada." (nota de campo de Lorena).

No obstante, Lleixà (2002) destaca que las adaptaciones curriculares son esenciales en las clases de EF multiculturales para fomentar la igualdad de oportunidades.

Este tipo de discursos dejan entrever cómo las posibles necesidades propias del alumnado inmigrante pasan desapercibidas por el profesorado de EF. Las diferencias culturales no se consideran tan relevantes como las diferencias motoras o perceptivas, las cuales sí se perciben y se atienden.

De acuerdo con esta percepción, el perfil "romántico-asimilador" señala que en sus sesiones es necesario elaborar ningún protocolo específico de acogida, puesto que son suficientes las dinámicas que se crean en clase: "La acogida, por la misma idiosincrasia de la asignatura, hace que no sea muy necesaria." (entrevista con Lucas).

De igual modo, tampoco se hace nada en concreto para favorecer la comunicación verbal con el alumnado puesto que las demostraciones prácticas de los ejercicios facilitan la comprensión de las tareas, según estos mismos: "Yo es que lo tengo muy fácil porque, aunque no me entiendan, como en EF es todo bastante visual, pues en principio no hay ningún problema." (entrevista con Mireia).

En esta línea, el perfil "romántico-asimilador" se muestra convencido de que la simple introducción de los juegos o danzas del mundo en las clases de EF es un ejemplo de la orientación intercultural de sus prácticas, aunque estas actividades destaquen por ser 
anecdóticas y más bien folclóricas (TORRES, 2008), y sin la implicación del propio alumnado, el cual puede no sentirse identificado con esos juegos si no ha pasado la infancia en su país de origen.

Por otra parte, desde este perfil se valora negativamente la calidad de la formación académica recibida. Destacan un exceso de enfoques teóricos descontextualizados y alejados de la realidad, y echan de menos la enseñanza de más estrategias prácticas a modo de recetas estandarizadas, ambas ideas criticadas por Grimminger y Möhwald (2016). Sin embargo, manifiestan tener altos niveles de satisfacción personal y profesional. Podría decirse que estas personas se describen como felices, contentas y orgullosas de ser las responsables de la EF.

En definitiva, el profesorado "romántico-asimilador" subraya de manera reiterada y convencida la total "normalidad" en que se desarrollan sus sesiones de EF en un contexto multicultural, obviándose así las necesidades educativas del alumnado inmigrante (problemas lingüísticos, baja motivación, choque cultural, etc.).

\subsection{EL PERFIL "QUEMADO-PREJUICIOSO"}

En la escuela multicultural existe otro perfil docente caracterizado por su pesimismo y su visión negativa sobre la escolarización del alumnado inmigrante. Es un perfil que atribuye su estado de estrés laboral a la presencia del colectivo inmigrante en la escuela, y sobre todo, en el aula ordinaria. Esto favorece la existencia de unas actitudes discriminatorias y xenófobas que influyen directamente en su labor profesional.

Todas estas características encajan con el concepto de burnout señalado por Moriana y Herruzo (2004), el cual se entiende como el cansancio emocional y/o el estrés del docente, generador de un alto nivel de tensión, que afecta negativamente, sobre todo, a su nivel psicológico y fisiológico. Como consecuencia, su desarrollo profesional se ven afectados negativamente.

Desde este perfil se considera que la presencia del alumnado inmigrante en el aula ordinaria, y no en EF, genera un gran número de inconvenientes, motivo por el cual manifiesta abiertamente su visión negativa (FLORES; PRAT; SOLER, 2017). No obstante, la mayor parte de los problemas a los que hacen referencia encajan en el denominado "factor educativo" descrito por Alegre (2008): a) la distribución desigual de la escolarización del alumnado inmigrante (formación de escuelas guetos); b) la falta de personal docente; c) el poco asesoramiento y ayudas por parte de las administraciones públicas; y d) las continuas entradas y salidas del alumnado debido a la "matrícula viva": "Tienes muchos más alumnos por clase y encima te bajan el sueldo. Tú vas absorbiendo pero llega un momento que no puedes más, que 'explotas'. Antes de navidades estás con una ansiedad y con un agotamiento psicológico, que si trabajaras en otro barrio no tendrías." (entrevista con Natalia).

De igual modo, se pone especial énfasis en los aspectos que dificultan la tarea educativa sin contrastar si estos aspectos son específicos del colectivo inmigrante o también se dan en otros colectivos, como el bajo nivel académico previo; y la poca atención familiar o la relación conflictiva con ellas. de 25! Los otros, cada uno venido en momentos diferentes, de lugares diferentes, 
con necesidades completamente diferentes. ¡Es para pegarse un tiro! (entrevista con Lorena).

A su vez, el discurso de este perfil reproduce muchos de los estereotipos y prejuicios existentes en la sociedad respecto a los diversos colectivos de inmigrantes, tanto a nivel cultural como de género; una visión discriminatoria ya constatada (PÀMIES, 2013). "La gente de Marruecos y toda esta zona son muy chulos y mentirosos" (entrevista con Lorena). Otra afirmación hacer acerca del tema es "Siempre hay alguna niña que no tiene tantas ganas de hacer la actividad deportiva. Pero lo encuentro normal porque a veces es por imposibilidad física, pues no tienen tanta habilidad que los chicos" (entrevista con Álvaro).

Bajo esta mirada negativa que configura el currículum oculto del profesorado, la intervención pedagógica de este colectivo se caracteriza por la reproducción continua de las tradicionales prácticas discriminatorias y estereotipadas que se desprenden de la herencia histórica de la EF (FLORES; PRAT; SOLER, 2015a).

Por otra parte, los resultados revelan que el desgaste profesional y personal puede provocar la resignación laboral, afectando así a la calidad de sus intervenciones educativas. "La profesora se describe como "una pasota". Según ella: "esto es debido a los muchos años de gritos y preocupaciones" [...]. "trabajar con este tipo de alumnado desgasta mucho" (nota de campo de Mar).

Su planificación curricular es rígida y desfasada, de modo que los contenidos y enfoques curriculares no se modifican con el paso de los años a pesar de los cambios en el currículum de EF. Generalmente mantiene una relación distante y fría con el alumnado, y se muestra indiferente hacia las necesidades sociales y económicas de las familias: "Yo soy profesora y no una asistenta social, o una monja de la caridad [...] no quiero profundizar en el origen de los problemas de mis alumnos." (nota de campo de Mar).

En el desarrollo de las clases, el castigo y la exclusión suelen ser estrategias frecuentes para incentivar al alumnado: "Mis castigos me funcionan porque yo juego con la ventaja de que mi asignatura motiva, y si no la hacen [clase de EF] les fastidio". (nota de campo de Lorena).

No obstante, los insultos, las agresiones o las situaciones racistas que puedan aparecer en la escuela se ignoran, se minimizan o se banalizan: "Alguna vez sí que se ha hecho algún insulto racista, pero yo no creo que sea tanto con conocimiento de causa [...] pues es lo primero que les aparece por la cabeza." (entrevista con Álvaro).

Considera que el alumnado debe aceptar los procesos de asimilación que el profesorado de EF promueve. "Tenemos una niña que viene con el pañuelo. Al igual que no dejo a los chavales que vengan con gorra, cuando vienen con el pañuelo se lo quitan obligatoriamente." (entrevista con Álvaro).

Coincidiendo con los otros dos perfiles, este profesorado también denuncia una deficitaria formación inicial respecto a la temática que aquí se aborda, e incluso la formación permanente puede ser menospreciada. "La formación permanente no puede ser obligatoria, sino opcional. Llega un momento que hay cinco conferencias y las cinco hablaban de lo mismo." (entrevista con Álvaro).

A diferencia de los perfiles anteriores, durante la trayectoria profesional de este perfil se han generado y acumulado sentimientos de frustración, desánimo y desgaste profesional 
y personal, provocando incluso que contemplen la posibilidad de cambiar de profesión: "Esta escuela te va chupando la sangre, te va gastando energías y te van saliendo arrugas y canas." (entrevista con Mar).

En definitiva, este perfil refleja un profesorado reticente en cuanto a la atención a la diversidad cultural se refiere, que incluso puede llegar a reconocer su falta de compromiso profesional.

\section{CONCLUSIONES}

En función de la percepción del profesorado de EF con más de cinco años de experiencia en una escuela multicultural sobre su alumnado inmigrante, su intervención pedagógica y su trayectoria profesional, en este trabajo se desprende una tipificación de tres perfiles pedagógicos de este colectivo.

El "sensible-inclusor" valora positivamente la presencia del alumnado inmigrante en el aula, y lo considera una oportunidad. Es capaz de captar las necesidades de su alumnado, las cuales suelen ser atendidas en cada sesión de EF con la utilización de un amplio repertorio de estrategias didácticas, siendo esto un ejemplo del tacto pedagógico que lo caracteriza. Además, cabe destacar su alta capacidad crítica y reflexiva, y su compromiso y preocupación por la mejora profesional para abordar mejor las relaciones interculturales.

El "romántico-asimilador" se caracteriza por no visualizar las problemáticas propias de la diversidad cultural debido a su amor incondicional hacia la EF y hacia sus supuestas ventajas para la inclusión. En consecuencia, su intervención educativa no se modifica en ningún momento ya que las necesidades educativas del alumnado pasan totalmente desapercibidas. En base a esta ausencia de crítica y reflexión, sus actuaciones poseen un carácter homogeneizador, lo cual es un ejemplo de los procesos de aculturación-asimilación existentes en sus sesiones de EF.

El "quemado-prejuicioso" es el que se resiste al enfoque de la EFI. A partir de un discurso confuso, este perfil asocia su estado de estrés laboral a la presencia del alumnado inmigrante en la escuela. Ante esta culpabilización directa, florecen actitudes xenófobas y racistas, las cuales posteriormente se trasladan a su intervención pedagógica en las sesiones de EF mediante la reproducción de las tradicionales prácticas discriminatorias y estereotipadas que se desprenden de la herencia histórica de la EF. En esta línea, este perfil expresa su rechazo hacia la interculturalidad y la atención a las necesidades educativas existentes.

Esta tipificación permite evitar generalizaciones y la homogenización del colectivo docente, de modo que refleja también la diversidad de posicionamientos existentes, así como la variedad de posturas, a menudo contradictorias y ambivalentes, que pueden darse en un mismo centro o colectivo. De igual modo, la descripción de estos tres perfiles puede ser clave a la hora de identificar o reconocer a qué perfil se aproxima más el profesorado de EF, para así poder abordar los puntos débiles y reforzar los fuertes, sobre todo en futuras investigaciones 0 en actividades de formación inicial y/o permanente del profesorado. Esto podría ser uno de los pasos previos para la posterior "verdadera" presencia de la EFI en la escuela del siglo XXI. 


\section{REFERENCIAS}

AGUADO, María Teresa. Pedagogía intercultural. Madrid: McGraw-Hill, 2003.

AGUADO, María Teresa; MATA, Patricia; GIL-JAURENA, Inés. Mobilizing intercultural education for equity and social justice. Time to react against the intolerable: A proposal from Spain.

Intercultural Education, v.28, n.4, p. 408-423, 2017.

ALEGRE, Miguel Ángel. Educación e inmigración. ¿Un binomio problemático? Revista de Educación, n. 345, p. 61-82, 2008.

BANKS, James. Handbook of research on multicultural education. New York: Macmillan, 1995.

BANKS, James; LYNCH, James. Multicultural education in western societies. London: Holt, Rinehart and Winston, 1986.

BARDIN, Laurence. El análisis de contenido. Madrid: Akal, 1986.

BARKER, Dean. In defence of white privilege: physical education teachers' understandings of their work in culturally diverse schools. Sport, Education and Society, Special Issue: Gender, Physical Education and Active Lifestyles, v. 24, n.2, p.134-146, 2017.

BENN, Tansin; DAGKAS, Symeon. Incompatible? compulsory mixed-sex physical education initial teacher training (PEITT) and the inclusion of Muslim women: a case-study on seeking solutions. European Physical Education Review, v. 12, n. 2, p. 181-200, 2006

BESALÚ, Xavier; LÓPEZ CUESTA, Begoña. Interculturalidad y ciudadanía: Red de escuelas interculturales. Las Rozas: Walters Kluwer, 2011.

BESALÚ, Xavier; TORT, Jaume. Escuela y sociedad multicultural: propuestas para trabajar con alumnado extranjero. Alcalá de Guadaira: Mad, 2009.

CAPLLONCH, Marta; GODALL, Teresa; LLEIXÀ, Teresa. El professorat d'educació física a l'escola multicultural. Percepcions del context i necessitats de formació. Temps d'educació, v. 33, p. 61-74, 2007.

CARBONELL, Francesc. Decálogo para una educación intercultural. Cuadernos De Pedagogía, n. 290, p. $90-94,2000$.

CARROLL, Bob; HOLLINSHEAD, Graeme. Etnhicity and conflict in physical education. British Educational Journal, v. 19, p.1, p. 59-76, 1993.

CARTER-THUILLIER, Bastian; LÓPEZ-PASTOR, Víctor Manuel; GALLARDO, Francisco. Inmigración, deporte y escuela. Revisión del estado de la cuestión. Retos. Nuevas tendencias en Educación Física, Deporte y Recreación, v. 32, p. 19-24, 2017.

CHEPYATOR-THOMSON, Jepkorir Rose; YOU, Jeongae; RUSELL, Jared. In service physical education teachers: Background and understanding of multicultural education. Journal of inService Education, v. 26, n. 3, p. 557-568, 2000.

CHOI, Wonseok; CHEPYATOR-THOMSON, Jepkorir. Rose. Multiculturalism in teaching physical education: a review of U.S. based literature. Journal of Research, v. 6, n. 2, p. 14-20, 2011. 
DAGKAS, Symeon. Exploring teaching practices in physical education with culturally diverse classes: a cross-cultural study. European Journal of Teaching Education, v. 30, n. 4, p. 431443, 2007.

\section{EUROPEAN STATISTICS. Population without the citizenship of the reporting country.} European Commission, 2017. Disponible en: http://ec.europa.eu/eurostat/tgm/graph. do?tab=graph\&plugin=1\&pcode=tps00157\&language=en\&toolbox=type. Acceso en: 22 dez 2017.

FERNÁNDEZ-BATANERO, José María; AGUILAR, José Manuel. Competencias docentes interculturales del profesorado de Educación Física en Andalucía (España). Movimento, v. 22, n. 3, p. 753-766, 2016.

FLECHA, Ramon; PUIGVERT, Lidia. Multiculturalismo y educación. In: LLEIXÀ, Teresa (ed.). Multiculturalismo y educación física. Barcelona: Paidotribo, 2002. p. 9-45.

FLINTOFF, Anne; FITZGERALD, Hayley; SCRATON, Sheila. The challenges of intersectionality: researching difference in physical education. International Studies in Sociology of Education, v. 18, n. 2, p. 73-85, 2008.

FLORES, Gonzalo; PRAT, Maria; SOLER, Susanna. La voz del profesorado de educación física sobre su formación acadèmica ante la realidad multicultural: anàlisis de la situación y propuestas de mejora. Revista Electrónica Interuniversitaria de Formación del Profesorado, v. 17, n. 2, p. 183-199, 2014.

FLORES, Gonzalo; PRAT, Maria; SOLER, Susanna. La intervención pedagógica del profesorado de educación física en un contexto multicultural: prácticas, reflexiones y orientaciones. Retos. Nuevas tendencias en Educación Física, Deporte y Recreación, n. 28, p. 248-255, $2015 a$.

FLORES, Gonzalo; PRAT, Maria; SOLER, Susanna. Echando la vista atrás: la voz del profesorado de Educación Física sobre su trayectoria profesional en una escuela multicultural. Apunts. Educación Física y Deportes, n. 12, p. 88-98, 2015b.

FLORES, Gonzalo; PRAT, Maria; SOLER, Susanna. La visión del profesorado de educación física sobre La presencia del alumnado inmigrante en la Escuela: ¿oportunidad o problema? Retos. Nuevas tendencias en Educación Física, Deporte y Recreación, n. 31, p. 64-68, 2017.

GARCÍA-FERNÁNDEZ, José Antonio; GOENECHEA, Cristina. Educación intercultural: análisis de la situación y propuestas de mejora. Barcelona: Wolters Kluwer España, 2009.

GARRETA, Jordi. La atención a la diversidad cultural en Cataluña: exclusión, segregación e interculturalidad. Revista de Educación, n. 335, p. 213-233, 2011.

GIROUX, Henry. Teachers as Transformative Intellectuals. Social Education, v. 49, n. 5, p. 376-379, 1985.

GOENECHEA, Cristina; GARCÍA-FERNÁNDEZ, José Antonio; JIMÉNEZ, Rafael. Los dilemas de la atención educativa a los alumnos inmigrantes recién llegados: Estudio comparativo de los modelos andaluz (ATAL) y madrileño (aulas de enlace). Profesorado. Revista de Currículum y Formación del Profesorado, v. 15, n. 3, p. 263-278, 2011.

GRIMMINGER, Elke. Intercultural competence among sports and PE teachers. Theoretical foundations and empirical verification. European Journal of Teacher Education, v. 34, n. 3, p. 317-331, 2011. 
GRIMMINGER, Elke; MÖHWALD, Aiko. Intercultural education in physical education: results of a quasi-experimental intervention study with secondary school students. Physical Education and Sport Pedagogy, n. 22, p. 445-458, 2016.

HEINEMANN, Klaus. Introducción a la metodología de la investigación empírica en las ciencias del deporte. Barcelona: Paidotribo, 2003.

INSTITUT D'ESTADÍSTICA DE CATALUNYA. Evolució de la població total i estrangera. 2000-2016. Generalitat de Catalunya, 2017. Disponible en: http://www.idescat.cat/ poblacioestrangera/?b=0\#Plegable. Acceso em: $10 \mathrm{dez} .2017$.

LINCOLN, Yvonna; GUBA, Egon. Naturalistic inquiry. Newbury Park, CA: Sage, 1985.

LÓPEZ-PASTOR, Víctor Manuel; PÉREZ, Ángel; MONJAS, Roberto. La atención a la diversidad en el área de educación física. La integración del alumnado con necesidades educativas específicas, especialmente el alumnado inmigrante y de minorías étnicas. Lecturas: Educación Física y Deportes, n. 106. 2007. Disponible en: https://www.efdeportes.com/efd106/la-atencion-a-ladiversidad-en-educacion-fisica.htm. Acceso en: 8 ago. 2017.

LÓPEZ-PASTOR, Víctor Manuel. Didáctica de la educación física, desigualdad y transformación social. Estudios Pedagógicos, n. 38, p. 155-176, 2012.

LÓPEZ-RODRÍGUEZ, Francesc; SENTÍS, Ferrán. Las adaptaciones del currículum en primaria. In: Alcudia, Rosa (ed.), Atención a la diversidad. Barcelona: Graó, 2000. p. 85-90.

LLEIXÀ, Teresa. Atención a la diversidad en el currículum de educación física. In: LLEIXÀ, Teresa (ed.) Multiculturalismo y educación física. Barcelona: Padiotribo, 2002. p. 79-111.

MARCELO, Carlos. El profesorado principiante: inserción a la docencia. Barcelona: Octaedro, 2008.

MORIANA, Juan Antonio; HERRUZO, Javier. Estrés y burnout en profesores. Internacional Journal of clinical and Health Psychology, v. 4, n. 3, p. 597-621, 2004.

OLLER, Carles; COLOMÉ, Eva. Alumnado de otras culturas: acogida y escolarización. Barcelona: Graó, 2010.

PÀMIES, Jordi. El impacto de los agrupamientos escolares. Los espacios de aprendizaje y sociabilidad de los jóvenes de origen marroquí en Barcelona. Revista de Educación, n. 363, p. 133-158, 2013.

PASTOR, Juan Carlos; GONZÁLEZ, Sixto; CUEVAS, Ricardo; GIL-MADRONA, Pedro. El profesorado de educación física ante la inmigración. Cuadernos de Psicología del Deporte, $v$. 10(Supl.), n. 2. p. 79-84, 2010.

PRAT, Maria. Deporte y ciudadanía. In: PUIG, Josep (org.), Entre otros: Compartir la educación para la ciudadanía. Barcelona: ICE-Horsori, 2010. p. 149-164.

SALES, Auxiliadora. La formació dels professionals en educació intercultural. In: Grup de Recerca en Educació Intercultural (ed.). Multiculturalitat, educació i societat. Palma de Mallorca: Universitat de les Illes Balears, Edicions UIB, 2007. p. 205-230.

SAMPÉ, Marc; ARANDIA, Maite; ELBOJ, Carmen. Actuaciones educativas que están consiguiendo éxito educativo en centros educativos con alumnado inmigrante. Revista Interuniverstaria de Formación del Profesorado, v. 26, n. 1, p. 119-132, 2012. 
SOLER, Susanna; FLORES, Gonzalo; PRAT, Maria. La educación física y el deporte como herramientas de inclusión de la población inmigrante en Cataluña: el papel de la escuela y la administración local. Pensar a Prática, v. 15, n. 1, p. 1-19, 2012.

SOLER, Susanna; ISPIZUA, Marian; MENDOZA, Nuria. Integración social y deporte. In: GARCÍA- FERRANDO, Manuel; PUIG, Núria, LAGARDERA, Francisco; LLOPIS, Ramón; VILANOVA, Anna (coord.), Sociología del deporte. 4.ed. Madrid: Alianza, 2017. p. 145-168.

TORRES, Jurjo. Diversidad cultural y contenidos escolares. Revista de Educación, n. 345, p. 83-110, 2008.

VAN MANEN, Max. El tacto en la enseñanza. El significado de la sensibilidad pedagógica. Barcelona: Paidós, 1998.

WEBER, Max. Economía y sociedad: esbozo de sociología comprensiva. 6. reimp. México: Fondo de Cultura Económica, 1983. 